OPEN ACCESS

Edited by:

Anna Onnis,

University of Siena, Italy

Reviewed by:

Alexander Grigoryevich

Tonevitsky,

National Research University Higher

School of Economics, Russia

Olof Gidlöf,

Lund University, Sweden

${ }^{*}$ Correspondence:

Juan M. Falcon-Perez

jfalcon@cicbiogune.es

tPresent address: Jose L. Lavin,

Applied Mathematics Department, Bioinformatics Unit NEIKER-(BRTA),

Bizkaia Technology Park, Bizkaia,

Spain

Marc Clos-Garcia,

Novo Nordisk Foundation Center for Basic Metabolic Research, Faculty

of Health and Medical Sciences,

University of Copenhagen

Copenhagen, Denmark

Specialty section This article was submitted to Membrane Traffic,

a section of the journal

Frontiers in Cell and Developmental

Biology

Received: 02 October 2020

Accepted: 07 December 2020

Published: 12 January 2021

\section{Extracellular Vesicles From Liver Progenitor Cells Downregulates Fibroblast Metabolic Activity and Increase the Expression of Immune-Response Related Molecules}

Felix Royo ${ }^{1,2}$, Mikel Azkargorta', Jose L. Lavin ${ }^{1+}$, Marc Clos-Garcia't, Ana R. Cortazar, ${ }^{1,3}$, Monika Gonzalez-Lopez'1, Laura Barcena', Hernando A. del Portillo ${ }^{4,5,6}$, María Yáñez-Mó7, Antonio Marcilla ${ }^{8,9}$, Francesc E. Borras ${ }^{10,11,12, ~ H e c t o r ~ P e i n a d o ~}{ }^{13}$, Isabel Guerrero ${ }^{14}$, Mar Váles-Gómez ${ }^{15}$, Unai Cereijo ${ }^{16}$, Teresa Sardon ${ }^{16}$, Ana M. Aransay 1,2, Felix Elortza ${ }^{1,2}$ and Juan M. Falcon-Perez ${ }^{1,2,17 *}$

\footnotetext{
${ }^{1}$ Center for Cooperative Research in Biosciences, Bizkaia Technology Park, Bizkaia, Spain, ${ }^{2}$ Centro de Investigación Biomédica en Red de Enfermedades Hepáticas y Digestivas, Madrid, Spain, ${ }^{3}$ Centro de Investigación Biomédica en Red de Cáncer, Instituto de Salud Carlos III, Madrid, Spain, ${ }^{4}$ ISGlobal, Hospital Clínic - Universitat de Barcelona, Barcelona, Spain, ${ }^{5}$ Health Sciences Research Institute Germans Trias i Pujol, Badalona, Spain, ${ }^{6}$ Institució Catalana de Recerca i Estudis Avançats, Barcelona, Spain, ' Departamento de Biología Molecular, Centro de Biología Molecular Severo Ochoa, Instituto de Investigaciones Sanitarias la Princesa, Universidad Autónoma de Madrid, Madrid, Spain, ${ }^{8}$ Àrea de Parasitologia, Departament de Farmàcia i Tecnologia Farmacèutica i Parasitologia, Universitat de València, Valencia, Spain, ${ }^{9}$ Joint Research Unit on Endocrinology, Nutrition and Clinical Dietetics, Health Research Institute La Fe, Universitat de València, Valencia, Spain, ${ }^{10}$ Department of Cell Biology, Physiology and Immunology, Autonomous University of Barcelona, Barcelona, Spain, ${ }^{11}$ REMAR-IVECAT Group- "Germans Trias i Pujol" Health Science Research Institute (IGTP), Badalona, Spain,

${ }^{12}$ Nephrology Department- "Germans Trias i Pujol” University Hospital, Can Ruti Campus, Badalona, Spain,

${ }^{13}$ Microenvironment and Metastasis Laboratory, Molecular Oncology Program, Spanish National Cancer Research Center, Madrid, Spain, ${ }^{14}$ Tissue and Organ Homeostasis, Centro de Biología Molecular "Severo Ochoa", Universidad Autónoma de Madrid, Madrid, Spain, ${ }^{15}$ Spanish National Centre for Biotechnology, Spanish National Research Council, Madrid, Spain,

${ }^{16}$ Anaxomics Biotech, Barcelona, Spain, ${ }^{17}$ Ikerbasque, Basque Foundation for Science, Bilbao, Spain
}

Extracellular vesicles (EVs) mediate cell-to-cell crosstalk whose content can induce changes in acceptor cells and their microenvironment. MLP29 cells are mouse liver progenitor cells that release EVs loaded with signaling cues that could affect cell fate. In the current work, we incubated 3T3-L1 mouse fibroblasts with MLP29-derived EVs, and then analyzed changes by proteomics and transcriptomics. Results showed a general downregulation of protein and transcript expression related to proliferative and metabolic routes dependent on TGF-beta. We also observed an increase in the ERBB2 interacting protein (ERBIN) and Cxcl2, together with an induction of ribosome biogenesis and interferon-related response molecules, suggesting the activation of immune system signaling.

Keywords: extracellular vesicles (EVs), exosomes, MLP29, fibroblast, cell crosstalk, immune response 


\section{INTRODUCTION}

Extracellular vesicles (EVs) play an important role in cellto-cell communication by interchanging messages between cells through their cargo and surface proteins (Fatima and Nawaz, 2017). Among their cargo, there are bioactive molecules capable of stimulating regenerative programs in damaged tissues (Fatima et al., 2017). In that regard, different types of EVs playing a role in liver regeneration and with antifibrotic effect have been described. Mesenchymal stem cell (MSC)-derived EVs mediate tissue regeneration in liver fibrosis (Katsuda and Ochiya, 2015; Damania et al., 2018). Moreover, they facilitate the recovery after ischemia in cardiac tissue, shortening the wound healing time, and reducing scar formation by modulating cellular migration, proliferation, and collagen synthesis (Zhao et al., 2015).

Liver stem cells constitute another population that secrete EVs with regenerative effects. Liver stem cell-derived EVs ameliorated renal function and morphology (Herrera Sanchez et al., 2014) and favor liver regeneration after hepatectomy in rats (Herrera et al., 2010) and after liver damage induced by non-alcoholic steatohepatitis (NASH; Bruno et al., 2020). They also show anti-tumoral effects (Brossa et al., 2020) and effectively reduce liver injury during hypoxic conditions created by normothermic machine liver perfusion associated to liver transplant, suggesting that liver stem cell-derived EVs can be employed to improve transplantation techniques (Rigo et al., 2018).

In the present work, we aimed to determine the effects of EVs released by MLP29, a cell line with characteristics of progenitor cell, isolated from fetal liver. During previous years, our group has characterized the proteome (Conde-Vancells et al., 2008), transcriptome (Royo et al., 2013), and metabolome (Royo et al., 2019b) of the EVs released by this cell line. Compared to EVs released by primary cultures of hepatocytes, MLP29-derived EVs are more resistant to mechanical stress (Royo et al., 2019b). Moreover, they can circulate in the organism to reach different organs, including liver, lung, spleen, and brain, according to studies of bio-distribution performed in vivo (Royo et al., 2019a). Given those characteristics, we hypothesize that MLP29-derived EVs are meant to have an effect over cellular parenchyma. To characterize the nature of such effect, we have chosen the 3T3L1 fibroblasts, a cell line that can be differentiated into adipocytes and also has the ability to produce collagen and, therefore, mimic a fibrotic response (Li et al., 2019). We describe the crosstalk observed between these cell lines, as it could give information about the existing cell-to-cell communication in the organism by progenitor cells and fibroblasts.

\section{MATERIALS AND METHODS}

\section{Cell Lines}

All media and reagents for tissue culture were purchased from GIBCO (Life Technologies Inc.). MLP29 is a murine liver progenitor cell line (Medico et al., 1996). The embryonic murine fibroblast cell line 3T3-L1 was purchased from ATCC (CL-173) as a murine embryonic fibroblast cell line.

\section{EVs Production and Purification}

MLP29 cells were plated in 150-mm dishes, at 10 million cells per dish. Cells were cultured in complete Dulbecco's modified Eagle medium medium [DMEM supplemented with $10 \%(\mathrm{v} / \mathrm{v})$ fetal bovine serum (FBS), $0.1 \mathrm{mg} / \mathrm{ml}$ streptomycin, and $100 \mathrm{units} / \mathrm{ml}$ penicillin (GIBCO, Life Technologies Inc.)] for $24 \mathrm{~h}$ at $37^{\circ} \mathrm{C}$ and $5 \%$ of $\mathrm{CO}_{2}$. Then, cells were washed twice with Dulbecco's modified phosphate-buffered saline (PBS) and incubated for $48 \mathrm{~h}$ (MLP29 cells) in $25 \mathrm{mM}$ HEPES-containing complete DMEM medium [contaminating vesicles were first removed by overnight centrifugation at $110,000 \times g$ (Thery et al., 2006)] After incubation, media were collected, and EVs were isolated as previously described (Conde-Vancells et al., 2008). Briefly, culture supernatant was centrifuged at $1500 \times g$ for $10 \mathrm{~min}$ to remove lifted cells and cellular debris. The resultant supernatant was centrifuged at $10,000 \times g$ and $100,000 \times g$ for 30 and $75 \mathrm{~min}$, respectively. The resulting pellet was suspended in PBS, pooled, and again centrifuged at $100,000 \times g$ for $75 \mathrm{~min}$. The final pellet of EVs was suspended in PBS and stored at $-80^{\circ} \mathrm{C}$. Morphology, quantification, and the presence of typical EV markers are presented in Supplementary Figure 1. As control, EV preparations were obtained from similar amount of media that have never been in contact with cells, to ensure that the observed effect was not due to the media.

\section{EV Treatment of 3T3-L1}

Approximately 2 million 3T3-L1 cells were treated with the EVs obtained from 200 million MLP29 cells. On average, this represents $20 \mu \mathrm{g}$ of protein (measured by Bradford) contained in $8.9^{*}$ E10 particles (measured with Nanosight LM10) per treatment. A total of six plates were treated, with three different preparations of EVs and three preparations of controls, for $24 \mathrm{~h}$, in $25 \mathrm{mM}$ HEPES-containing complete DMEM medium (vesicles from tissue culture media were first removed by overnight centrifugation at $110,000 \times g$ ). At the end of the incubation, plates were washed twice with PBS, detached with Tryple (GIBCO), and then divided equally for proteomic and transcriptomic analyses. An aliquot was employed to measure cell viability, which was higher than $90 \%$ in all the cases. Trypsin was removed after pelleting the cells $1000 \times \mathrm{xg}$ for $5 \mathrm{~min}$, which were frozen in dry ice and stored at $-80^{\circ} \mathrm{C}$ until processing.

\section{RNA Library and Sequencing}

Total RNA was extracted with RNeasy (Qiagen), including the step of DNase treatment for all the samples, and finally eluted in RNase-free water. The quantity and quality of the RNAs were evaluated using Qubit RNA HS Assay Kit (Life Technologies, Cat.\# 32855) and Agilent RNA Nano Chips (Agilent Technologies, Cat.\# 5067-1511), respectively.

Sequencing libraries were prepared using "TruSeq Stranded mRNA LT sample prep kit" (Illumina Inc., Cat.\# RS-1222101 or RS-122-2102), following the "TruSeq ${ }^{\circledR}$ Stranded mRNA Sample Preparation LS Protocol (Part \# 15031058 Rev. E). In brief, starting from $1000 \mathrm{ng}$ of total RNA, mRNA was purified, fragmented, and primed for cDNA synthesis. cDNA first strand was synthesized with SuperScript-II Reverse Transcriptase 
(Life Technologies, Cat.\#18064-014) for $10 \mathrm{~min}$ at $25^{\circ} \mathrm{C}, 15 \mathrm{~min}$ at $42^{\circ} \mathrm{C}, 15 \mathrm{~min}$ at $70^{\circ} \mathrm{C}$, and paused at $4^{\circ} \mathrm{C}$. cDNA second strand was synthesized with Illumina reagents at $16^{\circ} \mathrm{C}$ for $1 \mathrm{~h}$. Then, A-tailing and adaptor ligation were performed. Finally, enrichment of libraries was achieved by PCR $\left(30 \mathrm{~s}\right.$ at $98^{\circ} \mathrm{C} ; 15$ cycles of $10 \mathrm{~s}$ at $98^{\circ} \mathrm{C}, 30 \mathrm{~s}$ at $60^{\circ} \mathrm{C}, 30 \mathrm{~s}$ at $72^{\circ} \mathrm{C} ; 5 \mathrm{~min}$ at $72^{\circ} \mathrm{C}$ and paused at $4^{\circ} \mathrm{C}$ ). Libraries were quantified and visualized on an Agilent 2100 Bioanalyzer using Agilent High Sensitivity DNA kit (Agilent Technologies, Cat.\# G2938-90320) and Qubit dsDNA HS DNA Kit (Life Technologies, Cat.\# 32851) and single-read sequenced to obtain 30 million 50 -nt reads.

\section{Alignment and Quantification of Transcriptome}

Quality Control of sequenced samples was performed by FASTQC software. ${ }^{1}$ Reads were mapped against the mouse (mm10) reference genome by STAR (Dobin et al., 2013) program to account for spliced junctions. The resulting BAM alignment files for the samples were then used to generate a table of raw counts by Rsubread (Liao et al., 2013). Raw counts table was the input for the differential expression (DE) analysis, carried out by DESeq2 (Love et al., 2014), to detect differentially expressed genes among the different conditions.

\section{Proteomic Analysis and Quantification In Solution Digestion}

Samples were digested following the filter-aided FASP protocol described by Wisniewski et al. (2009) with minor modifications. Trypsin was added to a trypsin:protein ratio of $1: 10$, and the mixture was incubated overnight at $37^{\circ} \mathrm{C}$, dried out in a RVC2 25 speedvac concentrator (Christ), and resuspended in $0.1 \%$ formic acid (FA).

\section{Liquid Chromatography}

Liquid chromatography (LC) was performed using a NanoAcquity nano-HPLC (Waters), equipped with a Waters BEH C18 nano-column (200 mm $\times 75 \mu \mathrm{m} \mathrm{ID,} 1.8 \mu \mathrm{m})$. A chromatographic ramp of $120 \mathrm{~min}$ [5-60\% acetonitrile (ACN)] was used with a flow rate of $300 \mathrm{nl} / \mathrm{min}$. Mobile phase A was water containing $0.1 \% \mathrm{v} / \mathrm{v}$ formic acid, while mobile phase $\mathrm{B}$ was ACN containing $0.1 \% \mathrm{v} / \mathrm{v}$ FA; $0.5 \mu \mathrm{g}$ of each sample were loaded for each run.

\section{Mass Spectrometry}

Peptides were eluted directly into the LTQ Orbitrap XL mass spectrometer through a nanoelectrospray capillary source (Proxeon Biosystems), at $300 \mathrm{nl} / \mathrm{min}$ and using a 120-min linear gradient of $3-40 \% \mathrm{ACN}$, followed up by an increase to $40 \%$ ACN for the next $30 \mathrm{~min}$. The mass spectrometer automatically switched between MS and MS/MS acquisition in DDA mode. Full MS scan survey spectra (m/z 400-2,000) were acquired in the Orbitrap with mass resolution of 30,000 at $\mathrm{m} / \mathrm{z} 400$. After each survey scan, the six most intense ions above 1000 counts were sequentially subjected to collision-induced dissociation (CID) in the linear ion trap. Precursors with charge states of 2 and 3 were

${ }^{1}$ http://www.bioinformatics.babraham.ac.uk/projects/fastqc/ specifically selected for CID. Peptides were excluded from further analysis during $60 \mathrm{~s}$ using the dynamic exclusion feature.

\section{Database Search}

Database searching was performed using MASCOT 2.2.07 (Matrix Science, London, United Kingdom) against a UNIPROT/Swissprot database filled only with entries corresponding to Homo sapiens (without isoforms). For protein identification, the following parameters were adopted: carbamidomethylation of cysteines (C) as fixed modification, oxidation of methionines $(\mathrm{M})$ as variable modifications, $10 \mathrm{ppm}$ of peptide mass tolerance, $0.5 \mathrm{Da}$ fragment mass tolerance, and up to two missed cleavage points, peptide charges of +2 and +3 .

\section{Progenesis LC-MS Software Analysis for Label Free Differential Expression Analysis}

Progenesis LC-MS (version 2.0.5556.29015, Non-linear Dynamics) was used for the label-free differential protein expression analysis. One of the runs was used as the reference to which the precursor masses in all other samples were aligned to. Only features comprising charges of $2+$ and $3+$ were selected. The raw abundances of each feature were automatically normalized in a logarithmic scale against the reference run. Samples were grouped in accordance to the comparison being performed, and ANOVA analyses were performed. A peak list containing the information of all the features was generated and exported to the Mascot search engine (Matrix Science Ltd.). This file was searched against a UniProt/Swiss-Prot database under the conditions stated in the previous section, and the list of identified peptides was imported back to Progenesis LC-MS. Protein quantitation was performed based on the three most intense non-conflicting peptides (peptides occurring in only one protein), except for proteins with only two non-conflicting peptides. The significance of expression changes was tested at protein level, and proteins identified with at least two peptides and an ANOVA $p$ value $\leq 0.05$ were selected for further analyses.

\section{Annotation, Interaction, and Enrichment Pathway Analysis}

The list of transcripts and proteins was annotated using the functional annotation [selecting GO Molecular Function (MF), Biological Process (BP) Cellular Component (CC), KEGG, and REACTOME] using DAVID $6.8^{2}$ (Huang da et al., 2009) and later assigning them manually to different groups of interest according to keywords shown in Tables 1, 2, and avoiding redundancy. Afterward, the list of gene/proteins was loaded into Cytoscape v.3.8.0 (Shannon et al., 2003), and molecule interactions were retrieved from STRING App v.1.5.1 (Doncheva et al., 2019) with the confidence cutoff value of 0.4 and no addition of interacting molecules, against STRING experimental database, text-mining, and co-expression. Those nodes without edges were discharged, and the rest were clustered using the MCODE algorithms provided with clusterMaker2 v.1.3.1 (Morris et al., 2011). Finally, the clusters were manually annotated based

\footnotetext{
${ }^{2}$ https://david.ncifcrf.gov/

${ }^{3}$ https://cytoscape.org/
} 
TABLE 1 | Transcripts regulated in 3T3-L1 cells after EV treatment, classified by their involvement in different cellular processes, according to DAVID 6.8 annotation of each transcript.

\begin{tabular}{|c|c|}
\hline Process & Transcript \\
\hline \multicolumn{2}{|l|}{ Transcription } \\
\hline DNA binding & Dbp, Gm14288, Gm14420, Ctcfl, Gm14440 \\
\hline \multicolumn{2}{|l|}{ Translation } \\
\hline Proteasome & Shfm1, Ube2c \\
\hline Ribosome & Gm5148, Gm12191, Gm8430, Gm8430, Gm13826, Bc1 \\
\hline Mitochondria & Ppm1k \\
\hline \multicolumn{2}{|l|}{ Signaling } \\
\hline MAPK & Dusp6 \\
\hline EGF & Srf \\
\hline Chemokines & $\mathrm{CxCl12}$ \\
\hline ITFN & Ifit3, I830012016Rik, Ifi27 \\
\hline HIF & Serpine1 \\
\hline $\mathrm{NFKb}$ & Ptgs2 \\
\hline TGFb & Peg10 \\
\hline Cell adhesion & Zyx, Tnc, Prc1 \\
\hline Cell division & Bub1, Foxm1,Spry2, Ccnb1 \\
\hline Cytoskeleton & Tubb6, Mical2 \\
\hline
\end{tabular}

In red, upregulated transcripts; in black, downregulated genes.

on the enrichment groups obtained by functional enrichment analysis with STRING App v.1.5.1 (Doncheva et al., 2019) for each cluster (against GO Function, GO Components, GO Processes, Reactome pathways, and UniProt keywords) as shown in Figure 2. The functional pathway enrichment of Table 3 was performed using the Statistical Enrichment Test performed by Panther v.15.0 (Mi et al., 2019). A Fisher exact test was used in order to determine whether the proportion of genes considered into the GO term or categories differed significantly between the dataset and the background [ $p$ adjusted (FDR method) $<0.05$ ]. To determine the status of the pathway, fold change values were provided, and the category set employed was the GO Biological Processes.

\section{RESULTS}

\section{Transcriptomics}

The treatment of 3T3-L1 cells with MLP29-derived EVs induced a change in the expression of 45 transcripts above the significance threshold (see Figure 1A). Most of those changes were downregulating the expression of genes involved in many active processes of the cell. In Table 1, we show the main cellular processes affected by EV treatment, while in Supplementary Table 1, we show the differential gene expression fold changes and $p$ values. In addition, the data were deposited in the GEO repository under the access number GSE157022. A total of 45 transcripts were found significantly altered in EV-treated fibroblasts, and among them, 9 transcripts were upregulated. We found that Cxcl12 and Ifit3 were the most upregulated transcripts after treatment, while the predicted genes Gm19592

${ }^{4}$ http://www.pantherdb.org/
TABLE 2 | Proteins regulated in 3T3-L1 cells after EV treatment, classified according to their functional annotation in cellular processes, employing DAVID 6.8 database for the annotation of each protein.

\begin{tabular}{ll}
\hline Process & Proteins \\
\hline DNA replication & \\
Histone & H4C1 \\
Chromosomal segregation & ARL8A \\
Transcription & \\
RNA splicing & DCPS, DDX39A, EDC4, TARDBP, EFTUD2 \\
Ribonuclease inhibitor & ABCE1
\end{tabular}

Cytoskeleton

Anchoring of the basal body PPP2R1A, RAB11A

to the plasma membrane

Cadherin binding involved in $\quad$ ANXA1, CNN2, ENO1, HSP90AB1, EIF5, cell-cell adhesion RACK1, SEPTIN2, SNX9, SPTBN1, SPTAN1

Nuclear pore complex NUP155

Protein transport for nuclear signal IPO7, KPNB1

Actin filament formation $\quad$ ACTA2, CCT7

Focal adhesion $\quad$ FLNC, CFL1, DSTN, TPM4

Plasmatic membrane repair MYOF, TNS3

Remodeling cytoskeleton DPYSL3

Trafficking

Phagosome

AP2M1, COPB2

RAB7A, TUBB4B, TUBB5, ATP6V0D1, ATP6V1B2, ATP6V1E1

Apoptosis

Calcium ion binding

PDIA3, LMNA

Ion channel

ANXA4, ANXA6, LRP1, RCN1

CLIC4

\section{Protein metabolism}

Protein biosynthesis

Proteasome

EIF3M, ETF1, EIF5A, GARS1, HARS1, LARS1

PLAA, PSMC6, PSMC1, PSMA2, PSMA4, PSMB3, PSMD12, PSMD1

Protein degradation PREP, UBA1, UBA3, PPIA, IPO7, CAND1, ISG15, NEDD4

Ribosomal complex

HNRNPC, HNRNPM, EIF4A3, ILF3, PCBP2, RPL23A, RPLP2, RPS19, ATP5F1A

Protein folding/viral secretion PPIA

\section{Signaling}

Inhibitor Wnt

Insulin

G3BP1

ACACA, PRKAR2B, PRKACB, MAPK1, PCK2

Negative regulation of NF-kappaB ERBIN

Interferon

IFI204

Transcription inhibitor

BASP1

Carbon metabolism

Carbohydrate metabolism

Purine metabolism

ENO3, FH, PSAT1, SUCLA2, TKT

GAA, UGP2

NME2, PFAS

Lipid metabolism

ACSF2

VDAC1

AKR1B1, GLUD1, ETFB, GSTO1, MDH1, $\mathrm{MDH} 2, \mathrm{NSDHL}, \mathrm{PCYOX} 1, \mathrm{UGDH}$

In red, upregulated proteins; in black, downregulated ones.

and Gm19820 were the most downregulated. Furthermore, the most downregulated protein coding gene was Ptgs 2 , a gene involved in inflammation response. Some of the transcripts were assayed by qPCR, and we confirm the upregulation of Cxcl12 and Ifit 3 , as well as the downregulation of Tubb6 and the same trend 
TABLE 3 | Most significant enriched pathways according to Panther v.15.0 Enrichment Analysis (GO Biological Processes).

\begin{tabular}{|c|c|c|c|}
\hline Process & GO Term & Sign & Molecules \\
\hline $\begin{array}{l}\text { Negative regulation of transcription, } \\
\text { DNA-templated }\end{array}$ & GO:0045892 & Up & UBA3; Ifi27; Foxm1; Srf; BASP1; ILF3; NEDD4 \\
\hline Regulation of actin filament-based process & GO:0032970 & Down & CNN2; DSTN; ACTA2; CFL1; LRP1; SPTAN1; SPTBN1; SNX9 \\
\hline Ribosome biogenesis & GO:0042254 & Up & RPL23A; RPS19; ABCE1 \\
\hline Positive regulation of hydrolase activity & GO:0051345 & Down & PLAA; EIF5; RACK1; SNX9; HSP9OAB1 \\
\hline Cellular amino acid catabolic process & GO:0009063 & Down & GLUD1; PCYOX1 \\
\hline Regulation of phospholipase A2 activity & GO:0032429 & Down & ANXA1; PLAA; LRP1 \\
\hline Positive regulation of cell motility & GO:2000147 & Down & ANXA1; CXCl12; TKT; ACTA2; PLAA; CFL1; Spry2; LRP1 \\
\hline Positive regulation of innate immune response & GO:0045089 & Up & RPS19; IFI204 \\
\hline Cellular catabolic process & GO:0044248 & Down & $\begin{array}{l}\text { ISG15; PCK2; GLUD1; EDC4; PSMD12; PLAA; EIF4A3; PSMC6; LRP1; Ube2c; ETFB; } \\
\text { Dbp; PSMA4; GAA; PSMA2; PSMB3; RAB7A; ETF1; PSMD1; NEDD4; PCBP2; UBA1; } \\
\text { GSTO1; PCYOX1; DCPS }\end{array}$ \\
\hline Regulation of phagocytosis & GO:0050764 & Down & CNN2; LRP1; RACK1 \\
\hline Regulation of protein secretion & GO:0050708 & Down & ANXA1; PCK2; GLUD1; PPIA; LRP1; ANXA4; SPTBN1 \\
\hline G protein-coupled receptor signaling pathway & GO:0007186 & Down & ANXA1; LRP1; NME2; PRKACB \\
\hline Muscle structure development & GO:0061061 & Up & Srf; MYOF; FLNC; LMNA \\
\hline Inflammatory response & GO:0006954 & Down & ANXA1; PLAA; LRP1; Ptgs2 \\
\hline Regulation of focal adhesion assembly & GO:0051893 & Down & CFL1; LRP1 \\
\hline
\end{tabular}

The software defines that each pathway was upregulated (red) or downregulated (black) according to the fold change values of each gene/protein group and their function. For the molecules' annotation, red color indicates overexpression in fibroblast incubated with EVs, and black denotes downregulation with respect to untreated fibroblasts.
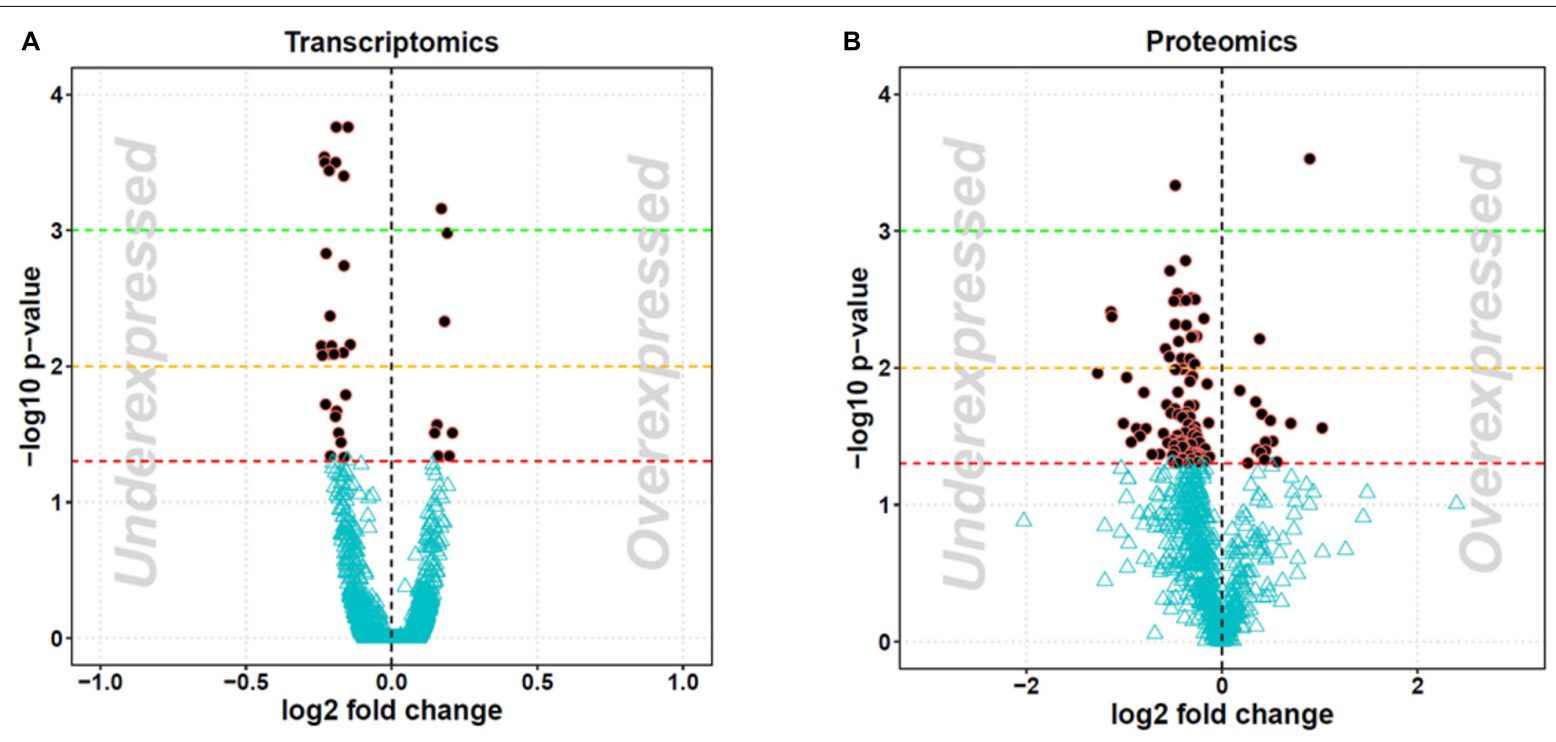

FIGURE 1 | Volcano plot representing the changes in 3T3-L1 cells after MLP29-derived EV treatment. The graphs show fold changes (log2 transformed) versus $p$ values (-log10 transformed). Significant regulated transcripts (A) and proteins (B) are represented as black dots in red circles. A gene or protein was considered regulated when its DE resulted in an adjusted $p$ value $<0.05$ (1.3 after -log10 transformation). Each experiment was performed in triplicate.

for Peg10, although in this case, the result was not significant (Supplementary Figure 3A).

\section{Proteomics}

The treatment of 3T3-L1 cells with MLP29-derived EVs induced a change in the expression of different proteins above the threshold of significance (see Figure 1B). Most of those changes were a protein expression downregulation. In Table 2, we show the annotation of proteins modified, while in Supplementary Table 2, we show the differential protein expression with fold changes and $p$ values. The expression of 107 proteins was affected by the EV treatment, and among them, 16 were upregulated, and the rest were downregulated after the treatment. The protein most over-represented was ERBIN, a protein that binds to the unphosphorylated form of the ERBB2 protein and regulates ERBB2 function and localization (Tao et al., 2014). The second most upregulated protein was ILF3, a protein with RNA binding capability involved in the formation of the ribosomal complex (Higuchi et al., 2012). Among the most downregulated proteins we found was RACK1, 
a scaffolding protein with participation in multiple functions such phagocytosis and cell-to-cell adhesion processes (Thorslund et al., 2011). The second most downregulated protein was $\mathrm{MDH} 2$, a mitochondrial malate dehydrogenase involved in oxredox processes (Minarik et al., 2002). We should mention that regarding fold change value, the most downregulated protein was GLUD1, a mitochondrial glutamate dehydrogenase also involved in catabolism and TCA cycle (Tzimagiorgis et al., 1993; Coloff et al., 2016).

At a molecular level, we did not observe any overlapping between regulated genes and proteins. However, regarding the process affected, we encountered some similarities between the routes affected according to transcriptomic and proteomic data. In both cases, we observed downregulation for the machinery related to control of cytoskeleton and cell motility. We also observed downregulation of major signaling cascades (i.e., MAPK1, NFKb, TGFb, and EGF) in both types of analyses, while some interferon-related molecules were upregulated. We had performed a qPCR to see if the regulation of the observed proteins may reflect on the transcriptome, and we have observed the increase of Myof transcript, as well as the downregulation of Plaa and Mdh1 transcripts, according to what was observed in the proteomics. However, this agreement was not clearly observed for Ccnb1 or Mapk1 (Supplementary Figure 3A). Interestingly, when 3T3 cells were treated with the vesicular fraction of the SEC-purified preparation of EVs (see Supplementary Materials and Methods), the downregulation of these last transcripts was observed (Supplementary Figure 3B), suggesting that some responses depend on the purity of the EV preparation, and to clearly visualize, one needs to remove protein aggregates that could unmask the response.

\section{Enrichment Analysis and Protein Networks}

To get a better insight of the general effect achieved by regulations of genes and proteins, in this section, the corresponding analysis was performed considering both types of molecules as a single set. As described in the "Materials and Methods" section, the enrichment analysis performed with the Panther v.15.0 platform ${ }^{5}$ (Mi et al., 2019) takes into account both the gene/protein regulated, as well as the direction of such regulation, and accordingly, it calculates the probability for the pathways stored in the Gene Ontology Biological Processes database to be upor downregulated. We performed this analysis with regulated genes/proteins, and the result is displayed in Table 3. The list includes a selection of pathways significantly regulated ( $p$ adjusted $<0.05$ ), manually curated for redundancy and for lack of cellular context. The results indicate a reduction in genes related to transcriptional activity in fibroblasts treated with MLP29derived EVs and upregulation of genes related to ribosome assembly, when compared to untreated cells. Interestingly, we also observed the upregulation in genes related to innate immunity. Some of the pathways that are downregulated included genes involved in migration, catabolism, inflammation, or phagocytosis.

\footnotetext{
${ }^{5} \mathrm{http}: / /$ www.pantherdb.org/
}

To further explore the relationship between regulated proteins/genes, we loaded all regulated molecules in the Cytoscape v.3.8.0 software (Shannon et al., 2003). We retrieved protein interactions from STRING App v.1.5.1 (Doncheva et al., 2019) as described in the section "Materials and Methods," and we discovered clusters of interacting molecules employing the MCODE algorithm from the clusterMaker2 App v.1.3.1 (Morris et al., 2011). Non-connected proteins were eliminated, and the annotations of the different clusters were decided according to the STRING functional enrichment annotations retrieved for each group of molecules, as well as protein individual annotation. Figure 2 illustrates the resulting interaction clusters, where the ribosome complex and the proteasome represent two welldefined groups. The machinery for mRNA splicing and different building blocks of the cytoskeleton appear as sub-groups within the largest cluster. Analysis in this interaction cluster showed that Cxcl2-Ptgs2 were related to regulation of immune activity and connected to other molecules related to cell migration. Other important groups were connected to cell cycling and the metabolism of carboxyl groups.

Regarding the functional meaning of the observed changes, the network presented in Figure 2 showed an increase in the presence of ribosome machinery, metabolism of fatty acids, and molecules related to interferon signaling. However, most of the cellular processes seemed to be inhibited, such as mRNA splicing, carbon metabolism, cell migration, the proteasome, and some molecules of the cytoskeleton. There was also a shortage in molecules involved in ATP hydrolysis. According to the results shown on the individual enrichment analysis, the interaction network suggested a slowdown of major cell activity, despite an increase of translation. We have observed as well a decrease in the metabolic activity measured by the MTT assay (Supplementary Materials and Methods and Supplementary Figure 2A) that could be explained by the downregulation described by the enrichment analysis.

\section{DISCUSSION}

In this work, we characterized the main effects produced by EVs derived from MLP29 liver progenitor cells in 3T3-L1 fibroblasts. The MLP29 cell line is an epithelial homogeneous cellular clone obtained by limiting dilution from a mouse embryonic liver cell line (Medico et al., 1996; Muller et al., 2002). This cell line responds to HGF with the whole array of its biological effects: scattering, survival, proliferation, and tubular morphogenesis (Medico et al., 1996). Moreover, their EVs carry a transcriptome enriched in signals regulating cell fate, proliferation, and cell organization (Royo et al., 2013). Accordingly, we analyzed the main changes in transcriptome and proteome in 3T3-L1 fibroblasts treated with MLP29-derived EVs. We should remark that purification of EVs through differential centrifugation could carry over other non-vesicular elements, which may also be responsible for the observed response, the reason why we have compared and further purified the preparation by SEC and observed a similar response to the fraction corresponding to vesicles. These results suggest that the 


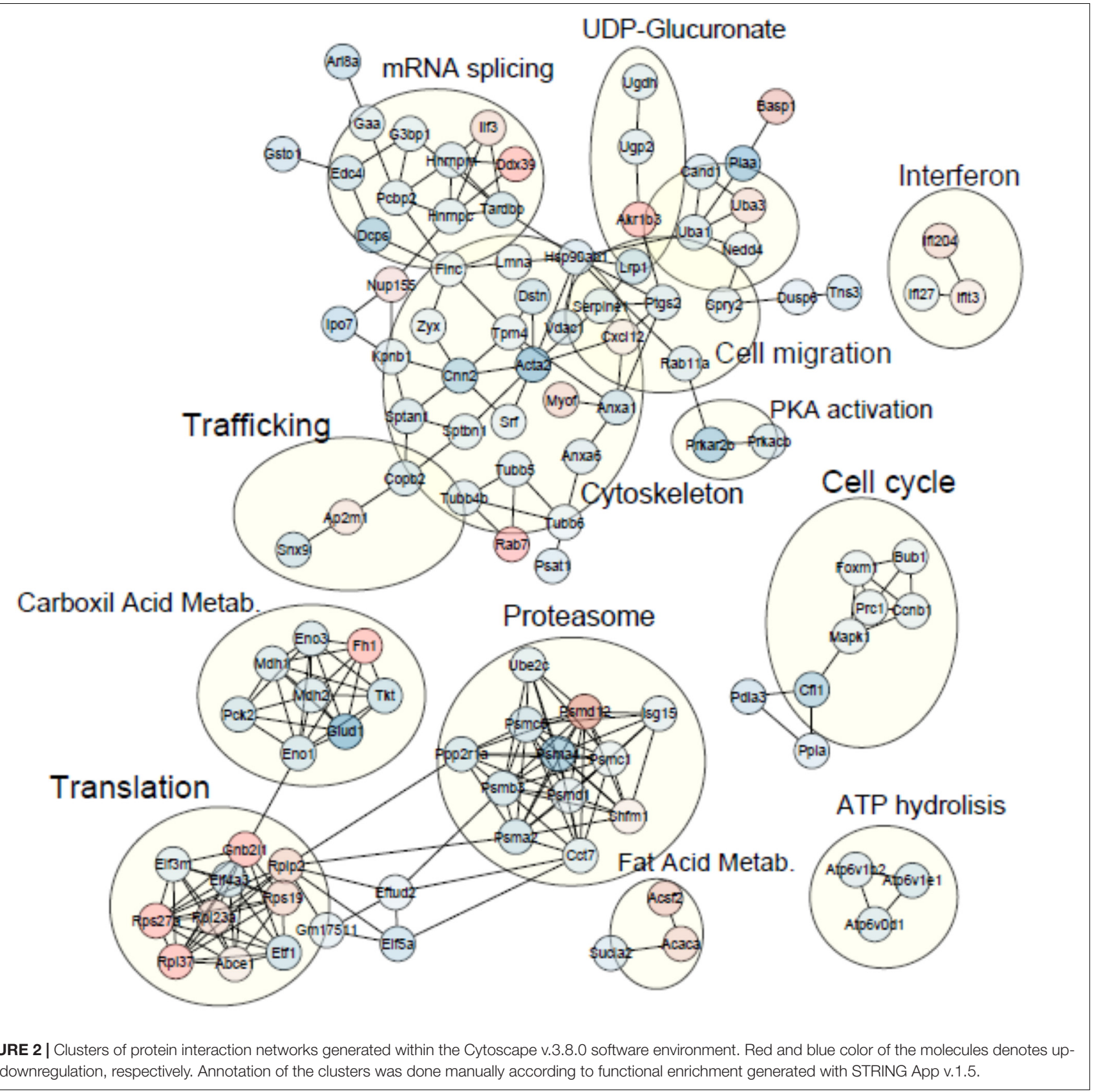

observed effects are mainly due the vesicular component of our preparations (Supplementary Figures 2B, 3B).

We observed that treatment with MLP29-derived EVs promoted a decrease in pathways related to catabolic activity, cell proliferation, and cytoskeleton remodeling. It should be highlighted that the protein most differentially expressed in treated cells is ERBIN, an ERBB2 interaction protein blocking ERK activation (Huang et al., 2003). ERBIN is an intracellular protein of the LAP family (LRR and PDZ), containing an LRR domain and a PDZ domain (Borg et al., 2000; Kolch, 2003), and it is believed to play a role in basolateral targeting in epithelial cells (Dillon et al., 2002). The mechanism for inhibition of the route of TGF- $\beta$ involves the formation of the ERBIN-MERLIN complex that disrupts the association between PAK2 and GTP-bound CDC42/RAC1. In fibroblasts, TGF- $\beta$ activates p21-activated kinase 2 (PACK2), which was activated in response to TGF- $\beta$ in different fibroblast cell lines, but not in epithelial cell lines (Wilkes et al., 2003; Wilkes et al., 2009). ERBIN also leads to deactivation of the ERK signal pathway, which is one of the main survival signaling pathways downstream HER2 (Yu et al., 2013).

We have also observed anti-apoptotic responses, such as the increase of the cytoskeleton protein myoferlin (MYOF), whose loss in fibroblasts is associated with a decrease in the expression of tight junction molecules and an increment in the number of cells positive for apoptotic markers (Leung et al., 2012). Taking into account that cells did not decrease their viability, 
the inhibitory responses observed do not lead to cell death. This agrees with previous studies of stem cell-derived EVs, showing the inhibition of fibrosis progression in models of kidney injury (Grange et al., 2019). Together, our results indicate that general cellular activity was slowed down in EV-treated compared to untreated fibroblasts, since transcription, cell motility, protein catabolism, and carbon metabolism were downregulated. Some enzymes with ATP hydrolytic activity were also downregulated. Consistently, we also detect reduction in metabolic activity measured by an MTT assay.

At the same time, there was an increase of signals related with immune system activation, centered in the activation of the transcription of the cytokine Cxcl12, a potent chemoattractant for hematopoietic cells. This molecule has been associated to fibrosis after radiation injury (Cao et al., 2019) and also to pro-metastatic stimulus of colon cancer cells through the PI3K/AKT/mTOR signaling pathway (Ma et al., 2017). However, in massive liver injury models where oval cell repair is involved, there is an upregulation of $\mathrm{Cxcl12}$ that interacts with oval cells because this cell type expresses CXCR4, the only known receptor for CXCL12 (Hatch et al., 2002).

The idea of a damage response is also reinforced by the observation of the increase of pathways related to ribosome biogenesis. These phenomena have been described to be associated to dsDNA sensing, a response to restrict virus reproduction and inflammatory response regulation (Bianco and Mohr, 2019). In this context, it is important to mention that MLP29-derived EVs contain dsDNA compatible with apoptotic features (Kruglik et al., 2019). Ribosome accumulation may also coordinate IFNB1 production, a pathway increased in fibroblasts that received MLP29-derived EVs. The upregulated molecules associated with the interferon pathway were Ifit3 and IFI204 (Bianco and Mohr, 2019). To conform to the hypothesis of ribosome accumulation, we measure the changes of transcriptional activity of $45 \mathrm{~S}$ pre-ribosomal RNA, observing a non-significant increase in cells treated by EVs by qPCR, and almost significant ( $p$ value 0.07 ) in cells treated with the vesicular fraction of our preparations (Supplementary Figure 3).

All the changes observed suggests that MLP29-derived EVs promoted somehow signals related to tissue damage in 3T3L1 fibroblasts that may be partially explained by the presence of dsDNA (Bianco and Mohr, 2019). The effects inferred from changes in proteins and genes indicate a slowdown in some general cell activities including cell motility and cytoskeleton remodeling, transcription, and protein degradation machinery, carbon metabolism and TCA processes, and the upregulation of molecules related to innate immune response. Moreover, the results offer a new confirmation of the role of EVs in the crosstalk between liver stem and stromal cells in the liver.

\section{REFERENCES}

Bianco, C., and Mohr, I. (2019). Ribosome biogenesis restricts innate immune responses to virus infection and DNA. eLife 8:e49551.

\section{DATA AVAILABILITY STATEMENT}

The datasets generated for this study can be found the NCBI GEO acession GSE157022.

\section{AUTHOR CONTRIBUTIONS}

HAP, MY-M, AM, HP, IG, FB, MV-G, and JF-P originally conceived and designed the study. FR, MA, MG-L, and LB performed the experiments. JL and AM performed the transcriptomics analysis. MA and FE performed the proteomics analysis. FR, MA, JL, MC-G, AC, UC, and TS performed the enrichment analysis and data visualization. All authors participated in data interpretation, discussion, and manuscript drafting and editing.

\section{FUNDING}

This work was supported by GEIVEX, Network of Excellence in the Research and Innovation on Exosomes REDIEX, SAF2015-71231-REDT, Network of Excellence in Translational NeTwork for the CLinical application of Extracellular VesicleS (TeNTaCLES), and RED2018-102411-T. The review is supported by Spanish Ministry of Science and Innovation, within the national Plan RTI2018-094969-B-I00, and Excellence Severo Ochoa grant Innovative Research Grant (SEV-2016-0644).

\section{ACKNOWLEDGMENTS}

We would like to acknowledge all the participants in the Practical Workshop Bioinformatics Tools to study exosomes' effects, organized by GEIVEX and REDIEX at CIC bioGUNE in November 2017.

\section{SUPPLEMENTARY MATERIAL}

The Supplementary Material for this article can be found online at: https://www.frontiersin.org/articles/10.3389/fcell.2020. 613583/full\#supplementary-material

Supplementary Figure 1 | Characterization of EVs derived from MLP29.

Supplementary Figure 2 | Measure of metabolic activity by MTT assay in 3T3.L1 cells after EV treatment.

Supplementary Figure 3 | Changes in the transcription of 3T3.L1 cells induced by EV treatment measure by qPCR.

Supplementary Table 1 | Significantly regulated transcripts.

Supplementary Table 2 | Significantly regulated proteins.

Borg, J. P., Marchetto, S., Le Bivic, A., Ollendorff, V., Jaulin-Bastard, F., Saito, H., et al. (2000). ERBIN: a basolateral PDZ protein that interacts with the mammalian ERBB2/HER2 receptor. Nat. Cell Biol. 2, 407-414. doi: 10.1038/ 35017038 
Brossa, A., Fonsato, V., Grange, C., Tritta, S., Tapparo, M., Calvetti, R., et al. (2020). Extracellular vesicles from human liver stem cells inhibit renal cancer stem cell-derived tumor growth in vitro and in vivo. Int. J. Cancer 147, 1694-1706. doi: 10.1002/ijc.32925

Bruno, S., Pasquino, C., Herrera Sanchez, M. B., Tapparo, M., Figliolini, F., Grange, C., et al. (2020). HLSC-derived extracellular vesicles attenuate liver fibrosis and inflammation in a murine model of non-alcoholic steatohepatitis. Mol. Ther. 28, 479-489. doi: 10.1016/j.ymthe.2019.10.016

Cao, J., Zhu, W., Yu, D., Pan, L., Zhong, L., Xiao, Y., et al. (2019). The involvement of SDF-1alpha/CXCR4 Axis in radiation-induced acute injury and fibrosis of skin. Radiat. Res. 192, 410-421. doi: 10.1667/rr15384.1

Coloff, J. L., Murphy, J. P., Braun, C. R., Harris, I. S., Shelton, L. M., Kami, K., et al. (2016). Differential glutamate metabolism in proliferating and quiescent mammary epithelial cells. Cell Metab. 23, 867-880. doi: 10.1016/j.cmet.2016. 03.016

Conde-Vancells, J., Rodriguez-Suarez, E., Embade, N., Gil, D., Matthiesen, R., Valle, M., et al. (2008). Characterization and comprehensive proteome profiling of exosomes secreted by hepatocytes. J. Proteome Res. 7, 5157-5166. doi: 10.1021/ pr8004887

Damania, A., Jaiman, D., Teotia, A. K., and Kumar, A. (2018). Mesenchymal stromal cell-derived exosome-rich fractionated secretome confers a hepatoprotective effect in liver injury. Stem Cell Res. Ther. 9:31.

Dillon, C., Creer, A., Kerr, K., Kumin, A., and Dickson, C. (2002). Basolateral targeting of ERBB2 is dependent on a novel bipartite juxtamembrane sorting signal but independent of the C-terminal ERBIN-binding domain. Mol. Cell Biol. 22, 6553-6563. doi: 10.1128/mcb.22.18.6553-6563. 2002

Dobin, A., Davis, C. A., Schlesinger, F., Drenkow, J., Zaleski, C., Jha, S., et al. (2013). STAR: ultrafast universal RNA-seq aligner. Bioinformatics 29, 15-21. doi: 10.1093/bioinformatics/bts635

Doncheva, N. T., Morris, J. H., Gorodkin, J., and Jensen, L. J. (2019). Cytoscape stringapp: network analysis and visualization of proteomics data. J. Proteome Res. 18, 623-632. doi: 10.1021/acs.jproteome.8b00702

Fatima, F., Ekstrom, K., Nazarenko, I., Maugeri, M., Valadi, H., Hill, A. F., et al. (2017). Non-coding RNAs in mesenchymal stem cell-derived extracellular vesicles: deciphering regulatory roles in stem cell potency. Inflammatory resolve, and tissue regeneration. Front. Genet. 8:161. doi: 10.3389/fgene.2017. 00161

Fatima, F., and Nawaz, M. (2017). Long distance metabolic regulation through adipose-derived circulating exosomal miRNAs: a trail for RNA-Based therapies? Front. Physiol. 8:545. doi: 10.3389/fphys.2017.00545

Grange, C., Tritta, S., Tapparo, M., Cedrino, M., Tetta, C., Camussi, G., et al. (2019). Stem cell-derived extracellular vesicles inhibit and revert fibrosis progression in a mouse model of diabetic nephropathy. Sci. Rep. 9:4468.

Hatch, H. M., Zheng, D., Jorgensen, M. L., and Petersen, B. E. (2002). SDF1alpha/CXCR4: a mechanism for hepatic oval cell activation and bone marrow stem cell recruitment to the injured liver of rats. Cloning Stem Cells 4, 339-351. doi: 10.1089/153623002321025014

Herrera, M. B., Fonsato, V., Gatti, S., Deregibus, M. C., Sordi, A., Cantarella, D., et al. (2010). Human liver stem cell-derived microvesicles accelerate hepatic regeneration in hepatectomized rats. J. Cell Mol. Med. 14, 1605-1618. doi: 10.1111/j.1582-4934.2009.00860.x

Herrera Sanchez, M. B., Bruno, S., Grange, C., Tapparo, M., Cantaluppi, V., Tetta, C., et al. (2014). Human liver stem cells and derived extracellular vesicles improve recovery in a murine model of acute kidney injury. Stem Cell Res. Ther. 5:124. doi: 10.1186/scrt514

Higuchi, T., Sakamoto, S., Kakinuma, Y., Kai, S., Yagyu, K., Todaka, H., et al. (2012). High expression of nuclear factor 90 (NF90) leads to mitochondrial degradation in skeletal and cardiac muscles. PLoS One 7:e43340. doi: 10.1371/journal.pone. 0043340

Huang, Y. Z., Zang, M., Xiong, W. C., Luo, Z., and Mei, L. (2003). Erbin suppresses the MAP kinase pathway. J. Biol. Chem. 278, 1108-1114. doi: 10.1074/jbc. $\mathrm{m} 205413200$

Huang da, W., Sherman, B. T., and Lempicki, R. A. (2009). Systematic and integrative analysis of large gene lists using DAVID bioinformatics resources. Nat. Protoc. 4, 44-57. doi: 10.1038/nprot.2008.211
Katsuda, T., and Ochiya, T. (2015). Molecular signatures of mesenchymal stem cell-derived extracellular vesicle-mediated tissue repair. Stem Cell Res. Ther. 6:212.

Kolch, W. (2003). Erbin: sorting out ErbB2 receptors or giving Ras a break? Sci STKE 2003:e37.

Kruglik, S. G., Royo, F., Guigner, J. M., Palomo, L., Seksek, O., Turpin, P. Y., et al. (2019). Raman tweezers microspectroscopy of circa $100 \mathrm{~nm}$ extracellular vesicles. Nanoscale 11, 1661-1679. doi: 10.1039/c8nr04677h

Leung, C., Shaheen, F., Bernatchez, P., and Hackett, T. L. (2012). Expression of myoferlin in human airway epithelium and its role in cell adhesion and zonula occludens-1 expression. PLoS One 7:e40478. doi: 10.1371/journal.pone. 0040478

Li, X. P., Liu, P., Li, Y. F., Zhang, G. L., Zeng, D. S., and Liu, D. L. (2019). LPS induces activation of the TLR4 pathway in fibroblasts and promotes skin scar formation through collagen I and TGF-beta in skin lesions. Int. J. Clin. Exp. Pathol. 12, 2121-2129.

Liao, Y., Smyth, G. K., and Shi, W. (2013). The Subread aligner: fast, accurate and scalable read mapping by seed-and-vote. Nucleic Acids Res. 41:e108. doi: 10.1093/nar/gkt214

Love, M. I., Huber, W., and Anders, S. (2014). Moderated estimation of fold change and dispersion for RNA-seq data with DESeq2. Genome Biol. 15:550.

Ma, J. C., Sun, X. W., Su, H., Chen, Q., Guo, T. K., Li, Y., et al. (2017). Fibroblastderived CXCL12/SDF-1alpha promotes CXCL6 secretion and co-operatively enhances metastatic potential through the PI3K/Akt/mTOR pathway in colon cancer. World J. Gastroenterol. 23, 5167-5178. doi: 10.3748/wjg.v23.i28. 5167

Medico, E., Mongiovi, A. M., Huff, J., Jelinek, M. A., Follenzi, A., Gaudino, G., et al. (1996). The tyrosine kinase receptors Ron and Sea control "scattering" and morphogenesis of liver progenitor cells in vitro. Mol. Biol. Cell 7, 495-504. doi: $10.1091 / \mathrm{mbc} .7 .4 .495$

Mi, H., Muruganujan, A., Ebert, D., Huang, X., and Thomas, P. D. (2019). PANTHER version 14: more genomes, a new PANTHER GO-slim and improvements in enrichment analysis tools. Nucleic Acids Res. 47, D419-D426.

Minarik, P., Tomaskova, N., Kollarova, M., and Antalik, M. (2002). Malate dehydrogenases-structure and function. Gen. Physiol. Biophys. 21, 257-265.

Morris, J. H., Apeltsin, L., Newman, A. M., Baumbach, J., Wittkop, T., Su, G., et al. (2011). clusterMaker: a multi-algorithm clustering plugin for Cytoscape. BMC Bioinformatics 12:436. doi: 10.1186/1471-2105-12-436

Muller, M., Morotti, A., and Ponzetto, C. (2002). Activation of NF-kappaB is essential for hepatocyte growth factor-mediated proliferation and tubulogenesis. Mol. Cell Biol. 22, 1060-1072. doi: 10.1128/mcb.22.4.1060-1072. 2002

Rigo, F., De Stefano, N., Navarro-Tableros, V., David, E., Rizza, G., Catalano, G., et al. (2018). Extracellular vesicles from human liver stem cells reduce injury in an Ex Vivo normothermic hypoxic rat liver perfusion model. Transplantation 102, e205-e210.

Royo, F., Cossio, U., Ruiz De Angulo, A., Llop, J., and Falcon-Perez, J. M. (2019a). Modification of the glycosylation of extracellular vesicles alters their biodistribution in mice. Nanoscale 11, 1531-1537. doi: 10.1039/c8nr0 $3900 \mathrm{c}$

Royo, F., Gil-Carton, D., Gonzalez, E., Mleczko, J., Palomo, L., Perez-Cormenzana, M., et al. (2019b). Differences in the metabolite composition and mechanical properties of extracellular vesicles secreted by hepatic cellular models. J. Extracell Vesicles 8:1575678. doi: 10.1080/20013078.2019.1575678

Royo, F., Schlangen, K., Palomo, L., Gonzalez, E., Conde-Vancells, J., Berisa, A., et al. (2013). Transcriptome of extracellular vesicles released by hepatocytes. PLoS One 8:e68693. doi: 10.1371/journal.pone.0068693

Shannon, P., Markiel, A., Ozier, O., Baliga, N. S., Wang, J. T., Ramage, D., et al. (2003). Cytoscape: a software environment for integrated models of biomolecular interaction networks. Genome Res. 13, 2498-2504. doi: 10.1101/ gr.1239303

Tao, Y., Shen, C., Luo, S., Traore, W., Marchetto, S., Santoni, M. J., et al. (2014). Role of Erbin in ErbB2-dependent breast tumor growth. Proc. Natl. Acad. Sci. U.S.A. 111, E4429-E4438. 
Thery, C., Amigorena, S., Raposo, G., and Clayton, A. (2006). Isolation and characterization of exosomes from cell culture supernatants and biological fluids. Curr. Protoc. Cell Biol. Chapter 3:Unit 3.22.

Thorslund, S. E., Edgren, T., Pettersson, J., Nordfelth, R., Sellin, M. E., Ivanova, E., et al. (2011). The RACK1 signaling scaffold protein selectively interacts with Yersinia pseudotuberculosis virulence function. PLoS One 6:e16784. doi: 10.1371/journal.pone.0016784

Tzimagiorgis, G., Leversha, M. A., Chroniary, K., Goulielmos, G., Sargent, C. A., Ferguson-Smith, M., et al. (1993). Structure and expression analysis of a member of the human glutamate dehydrogenase (GLUD) gene family mapped to chromosome 10p11.2. Hum. Genet. 91, 433-438.

Wilkes, M. C., Murphy, S. J., Garamszegi, N., and Leof, E. B. (2003). Cell-typespecific activation of PAK2 by transforming growth factor beta independent of Smad2 and Smad3. Mol. Cell Biol. 23, 8878-8889. doi: 10.1128/mcb.23.23.88788889.2003

Wilkes, M. C., Repellin, C. E., Hong, M., Bracamonte, M., Penheiter, S. G., Borg, J. P., et al. (2009). Erbin and the NF2 tumor suppressor Merlin cooperatively regulate cell-type-specific activation of PAK2 by TGF-beta. Dev. Cell 16, 433444. doi: 10.1016/j.devcel.2009.01.009

Wisniewski, J. R., Zougman, A., Nagaraj, N., and Mann, M. (2009). Universal sample preparation method for proteome analysis. Nat. Methods 6, 359-362.

Yu, D., Li, M., Ni, B., Kong, J., and Zhang, Z. (2013). Induction of neuronal mitophagy in acute spinal cord injury in rats. Neurotox Res. 24, 512-522. doi: 10.1007/s12640-013-9397-0
Zhao, Y., Sun, X., Cao, W., Ma, J., Sun, L., Qian, H., et al. (2015). Exosomes derived from human umbilical cord mesenchymal stem cells relieve acute myocardial ischemic injury. Stem Cells Int. 2015:761643.

Conflict of Interest: The authors declare that the research was conducted in the absence of any commercial or financial relationships that could be construed as a potential conflict of interest.

Citation: Royo F, Azkargorta M, Lavin JL, Clos-Garcia M, Cortazar AR, GonzalezLopez M, Barcena L, del Portillo HA, Yáñez-Mó M, Marcilla A, Borras FE, Peinado H, Guerrero I, Váles-Gómez M, Cereijo U, Sardon T, Aransay AM, Elortza F and Falcon-Perez JM (2021) Extracellular Vesicles From Liver Progenitor Cells Downregulates Fibroblast Metabolic Activity and Increase the Expression of Immune-Response Related Molecules. Front. Cell Dev. Biol. 8:613583. doi: $10.3389 /$ fcell.2020.613583

Copyright (C) 2021 Royo, Azkargorta, Lavin, Clos-Garcia, Cortazar, Gonzalez-Lopez, Barcena, del Portillo, Yáñez-Mó, Marcilla, Borras, Peinado, Guerrero, Váles-Gómez, Cereijo, Sardon, Aransay, Elortza and Falcon-Perez. This is an open-access article distributed under the terms of the Creative Commons Attribution License (CC BY). The use, distribution or reproduction in other forums is permitted, provided the original author(s) and the copyright owner(s) are credited and that the original publication in this journal is cited, in accordance with accepted academic practice. No use, distribution or reproduction is permitted which does not comply with these terms. 